\title{
Candida dubliniensis Identification in Brazilian Yeast Stock Collection
}

\author{
Priscilla de Laet Sant'Ana Mariano, Eveline Pípolo Milan/*, Daniel Archimedes da Matta, \\ Arnaldo Lopes Colombo/ ${ }^{+}$
}

\begin{abstract}
Laboratório Especial de Micologia, Disciplina de Doenças Infecciosas e Parasitárias, Universidade Federal de São Paulo, Rua Botucatu 740, 04023-062 São Paulo, SP, Brasil *Departamento de Microbiologia e Parasitologia, Universidade Federal do Rio Grande do Norte, Natal, RN, Brasil
\end{abstract}

\begin{abstract}
We investigated the presence of Candida dubliniensis among isolates previously identified as Candida albicans and maintained in a yeast stock collection from 1994 to 2000. All isolates were serotyped and further evaluated for antifungal susceptibility profile. After doing a screening test for $\mathrm{C}$. dubliniensis isolates based on the capability of colonies to grow at $42^{\circ} \mathrm{C}$, its final identification was obtained by randomly amplified polymorphic DNA (RAPD) analysis using three different primers. A total of 46 out of 548 screened isolates did not exhibit growth at $42^{\circ} \mathrm{C}$ and were further genotyped by RAPD. Eleven isolates were identified as $\mathrm{C}$. dubliniensis with RAPD analysis. Regarding serotypes, $81.5 \%$ of C. albicans and all C. dubliniensis isolates belonged to serotype A. Of note, 9 out of $11 \mathrm{C}$. dubliniensis isolates were obtained from patients with acquired immunodeficiency syndrome (Aids) and all of them were susceptible to azoles and amphotericin B. We found 17 (3\%) C. albicans isolates that were dose-dependent susceptibility or resistant to azoles. In conclusion, we found a low rate of C. dubliniensis isolates among stock cultures of yeasts previously identified as $\mathrm{C}$. albicans. Most of these isolates were recovered from oral samples of Aids patients and exhibited high susceptibility to amphotericin B and azoles. C. albicans serotype A susceptible to all antifungal drugs is the major phenotype found in our stock culture.
\end{abstract}

Key words: Candida dubliniensis - oral candidiasis - Candida spp. - Aids

Recently, Sullivan et al. (1995) described the novel species Candida dubliniensis, primarily associated with oral candidosis in human immunodeficiency virus (HIV) infected and acquired immunodeficiency syndrome (Aids) patients. C. dubliniensis shares many phenotypic characteristics with Candida albicans isolates and molecular methods are needed to distinguish both species (Sullivan et al. 1995, Coleman et al. 1997, Kirkpatrick et al. 1998, Sullivan \& Coleman 1998). Probably, this new species has been present in the community for a long period of time and has been misidentified as C. albicans (Schorling et al. 2000). Definitive identification of C. dubliniensis is a problem in routine diagnostic laboratories and surveillance studies performed by reference laboratories are necessary to better understand the epidemiology of this new species.

There are currently a wide variety of molecular techniques capable of identifying $C$. dubliniensis, including: DNA fingerprinting techniques using restriction endonuclease (REA) analysis, pulsed field gel electrophoresis-based methods, DNA fingerprinting using specific probes and polymerase chain reaction (PCR) based methods. Unfortunately, these methods are labor-intensive, ex-

This study was sponsored by Fundação de Amparo a Pesquisa do Estado de São Paulo, grants 99/ 07182-8 and 99/07134-3.

${ }^{+}$Corresponding author. Fax: +55-11-5549.6585. E-mail: colomboal@terra.com.br

Received 20 September 2002

Accepted 10 March 2003 pensive, and not readily applicable to routine use for identification purposes in most clinical microbiology diagnostic laboratories (Mannarelli \& Kurtzman 1998, Kurzai et al. 1999, Sullivan et al. 1999, Park et al. 2000).

In Brazil and South America there are a small number of reports addressing the epidemiology of this emergent species. The aims of this study were to identify $C$. dubliniensis strains among isolates from a large yeast culture collection including strains that were previously identified as $C$. albicans and to evaluate the serotypes and susceptibility profiles of isolates of both species.

\section{MATERIALS AND METHODS}

Samples - The isolates tested were selected among strains previously identified as C. albicans and maintained at the yeast stock collection of the Special Mycology Laboratory, Universidade Federal de São Paulo, between 1994 and 2000 . The samples had been stored at $-70^{\circ} \mathrm{C}$ in $10 \%$ glycerol, for different periods of time. We included strains isolated from different body fluids of HIV positive and negative patients as follow: blood, urine, esophageal and oropharyngeal samples.

C. albicans identification - All isolates were inoculated on CHROMagar Candida medium (CHROMagar Microbiology, Paris, France) and incubated at $30^{\circ} \mathrm{C}$ for up to 5 days with daily examination looking for light green colonies (typical of C. albicans) and dark green colonies (typical of C. dubliniensis). Representative light green and/or dark green colonies were further purified by subculture on Sabouraud dextrose agar (SDA, Difco Laboratories, Detroit, MI) and were tested for their ability to produce chlamydoconidia on corn meal Tween 80 agar (Kirkpatrick et al. 1998, Odds et al. 1998). 
Serotyping assay - All strains were prepared for slide agglutination serotyping with serodiagnostic reagent number 6 from Candida Check kit (Iatron Laboratories, Inc., Higashi-Kanda, Chiyoda, Tokio, Japan), as suggested in the package insert. C. albicans cultures were incubated on Sabouraud-dextrose agar plates for $48 \mathrm{~h}$ at $25^{\circ} \mathrm{C}$. Small amounts of specimen yeast cells were inoculated onto a Candida Check test tray and approximately $0.05 \mathrm{ml}$ of specific serum (number 6) was added for testing and physiological saline was added as control. The glass test tray was stirred for about 1-2 min. A positive agglutination reaction was interpreted by the visualization of aggregates considered to be indicative of C. albicans serotype A. Negative results in the agglutination reaction with reagent number 6 were considered to be indicative of $C$. albicans serotype B (Sullivan et al. 1995).

C. dubliniensis screening test - Following isolation on CHROMagar medium, a small portion of a single colony of each isolate was streaked for subculture over the surface on SDA agar plate and incubated at $42^{\circ} \mathrm{C}$ as previously described. All isolates that grew poorly or did not grow at all when incubated at $42^{\circ} \mathrm{C}$ were further genotyped by RAPD assays (Gales et al. 1998, Pinjon et al. 1998).

RAPD analysis - Template DNA for RAPD analysis was prepared from yeast cells grown in $2 \mathrm{ml}$ of YPD medium for $16 \mathrm{~h}$ at $30^{\circ} \mathrm{C}$ with agitation $(220$ r.p.m.) or until a density of $2 \times 10^{8}$ cells $/ \mathrm{ml}$ be achieved. DNA was extracted using a rapid, small-scale isolation protocol described previously (Wash et al. 1994). RNA was removed by treatment with RNase A (Amersham Pharmacia Biotech, Piscataway, NJ, USA) for $1 \mathrm{~h}$ at $37^{\circ} \mathrm{C}$. DNA concentration and purity were determined by optical density at $260 \mathrm{~nm}$ and ratio O.D. $260 \mathrm{~nm} / 280 \mathrm{~nm}$ determinations, respectively. RAPD tests were also performed with template DNA from reference strains of $C$. albicans (ATCC 76615) and $C$. dubliniensis (CBS 7987). RAPD analysis was performed as described previously with the oligonucleotide primer CDU (5' GCG ATC CCC A 3') (Sullivan et al. 1995), M2 (5' CTT GAT TGC C 3') and the oligonucleotide primer B-14 (5' GAT CAA GTC C 3'), the latter previously reported by Bauer et al. (1993). Aliquots of $40 \mathrm{ng}$ of total DNA were added to a $25 \mu 1$ reaction containing $2.5 \mu 1$ of a $10 \times$ PCR buffer (100 mM Tris-HCl, pH 8.3, $500 \mathrm{mM} \mathrm{KCl,} 3.5 \mathrm{mM}$ $\left.\mathrm{MgCl}_{2}\right), 4 \mu \mathrm{l}$ of dNTP mix (1.25 mM each dNTP), $0.4 \mu \mathrm{M}$ of primer, $0.5 \%$ (vol/vol) Tween 20 and 1.0 unit of Taq DNA polymerase (Amersham Pharmacia Biotech). Cycle conditions were $94^{\circ} \mathrm{C}$ for $1 \mathrm{~min}, 40^{\circ} \mathrm{C}$ for $1 \mathrm{~min}, 72^{\circ} \mathrm{C}$ for $2 \mathrm{~min}$, for 45 cycles and a final extension at $72^{\circ} \mathrm{C}$ for $10 \mathrm{~min}$, in a Perkin-Elmer DNA thermocycler (Gene Amp PCR System 9600). RAPD products were separated by electrophoresis for $3 \mathrm{~h}$ at $100 \mathrm{~V}$ in $1.5 \%(\mathrm{wt} / \mathrm{vol})$ agarose gels using Tris-Acetate-EDTA (TAE) buffer. Following electrophoresis, gels were stained with a solution containing $0.5 \mu \mathrm{g} / \mathrm{ml}$ ethidium bromide for $10 \mathrm{~min}$ and destained twice for 15 min in $300 \mathrm{ml}$ of distilled water. The gels were observed on UV transilluminator and photographed. RAPD profiles were defined with visual analysis, comparing the bands derived of the reference strains and the samples testing.

Susceptibility testing - All isolates were tested for susceptibility to amphotericin B, fluconazole, itraconazole and ketoconazole by using a broth microdilution assay performed in accordance with the NCCLS M27-A standard (NCCLS 1997). Reference grade powders of fluconazole (Pfizer Inc., New York, NY, USA), itraconazole (Janssen Pharmaceutical, Titusville, New Jersey, NY, USA), ketoconazole (Janssen Pharmaceutical) and amphotericin B (Sigma Chemical Corporation, Saint Louis, MO, USA) were used to obtain final drug dilutions ranging from 0.125 to $64 \mu \mathrm{g} / \mathrm{l}, 0.007$ to $4 \mu \mathrm{g} / \mathrm{ml}, 0.015$ to $8 \mu \mathrm{g} / \mathrm{ml}$ and 0.03 to 16 $\mu \mathrm{g} / \mathrm{ml}$, respectively. Briefly, broth microdilution testing was performed in sterile, flat-bottom 96-well microplates (Costar, Corning Incorporated, New York, USA), with RPMI-1640 (Angus Buffers \& Biochemicals, Niagara Falls, NY, USA) with L-glutamine, without bicarbonate, and buffered with MOPS at $\mathrm{pH}$ 7.0. The microplates containing the double of the final concentration of azoles were made in advance and stored at $-70^{\circ} \mathrm{C}$ for no more than three weeks until use. On the test day, the inoculum suspension had a turbidity adjusted by the spectrophotometer to that produced by a $0.5 \mathrm{McF}$ arland standard at $530 \mathrm{~nm}$ wavelength. A volume of $100 \mu$ l of the adjusted inoculum suspension was dispensed in each well resulting in the desired final drug concentration and inoculum size between 0.5 and $2.5 \times 10^{3}$ cells $/ \mathrm{ml}$. The plates were incubated at $35^{\circ} \mathrm{C}$ for $48 \mathrm{~h}$. Quality control strain $(C$. parapsilosis ATCC 22019) was included on each day of the assay to check the accuracy of the drug dilutions and the reproducibility of the results.

The Minimal Inhibitory Concentration (MIC) of amphotericin $\mathrm{B}$ was defined as the lowest concentration able to inhibit any visual growth. The MIC of azoles was the lowest concentration that exhibited prominent reduction in the turbidity, when compared with the growth control (drug-free) well (NCCLS 1997).

Breakpoint definitions for fluconazole and itraconazole MICs were those proposed by the NCCLS (1997). Due to the lack of consensual definitions of breakpoints for ketoconazole and amphotericin B MICs, arbitrary values were established, following those suggested in previous studies (Rodriguez-Tudela et al. 1995, St-Germain et al. 1995, Milan et al. 1998).

\section{RESULTS}

A total of 548 isolates previously identified as $C$. albicans on the bases of phenotypic characterization were considered for analysis. Among the 548 samples tested on CHROMagar Candida medium, only 10 exhibited a dark green colony color while the others exhibited a common light green colony color. All of them produced chlamydoconidia on corn meal Tween 80 agar. The clinical sources of the isolates evaluated were: blood (100), urine (86), esophageal (97), and oropharyngeal samples (265). All blood and urine isolates were obtained from HIV negative critical care patients. All esophageal samples and $50 \%$ of the oropharyngeal isolates were obtained from HIV positive patients.

After doing the temperature growth test, 46 isolates grew very slowly or not at all in these conditions and were further subjected to the RAPD analysis using three different primers. Based on RAPD findings, we concluded that 11 isolates exhibited the same profile of the CBS 7987 C. dubliniensis control organism. Among these $11 C$. 
dubliniensis isolates found, only 2 exhibited a typical dark green colony color on CHROMagar Candida medium.

Fig. 1 illustrates the RAPD banding patterns of the 11 clinical isolates obtained with RAPD using the 3 primers tested. Using the primer CDU, C. dubliniensis and $C$. albicans isolates yielded RAPD profiles with 7 and 6 strong bands, respectively, with molecular sizes ranging from 300 to $1300 \mathrm{bp}$. (Fig. 1A). The RAPD assay with primer B-14 yielded 6 strong bands to $C$. dubliniensis and 5 to $C$. albicans, with molecular sizes ranging from 400 to
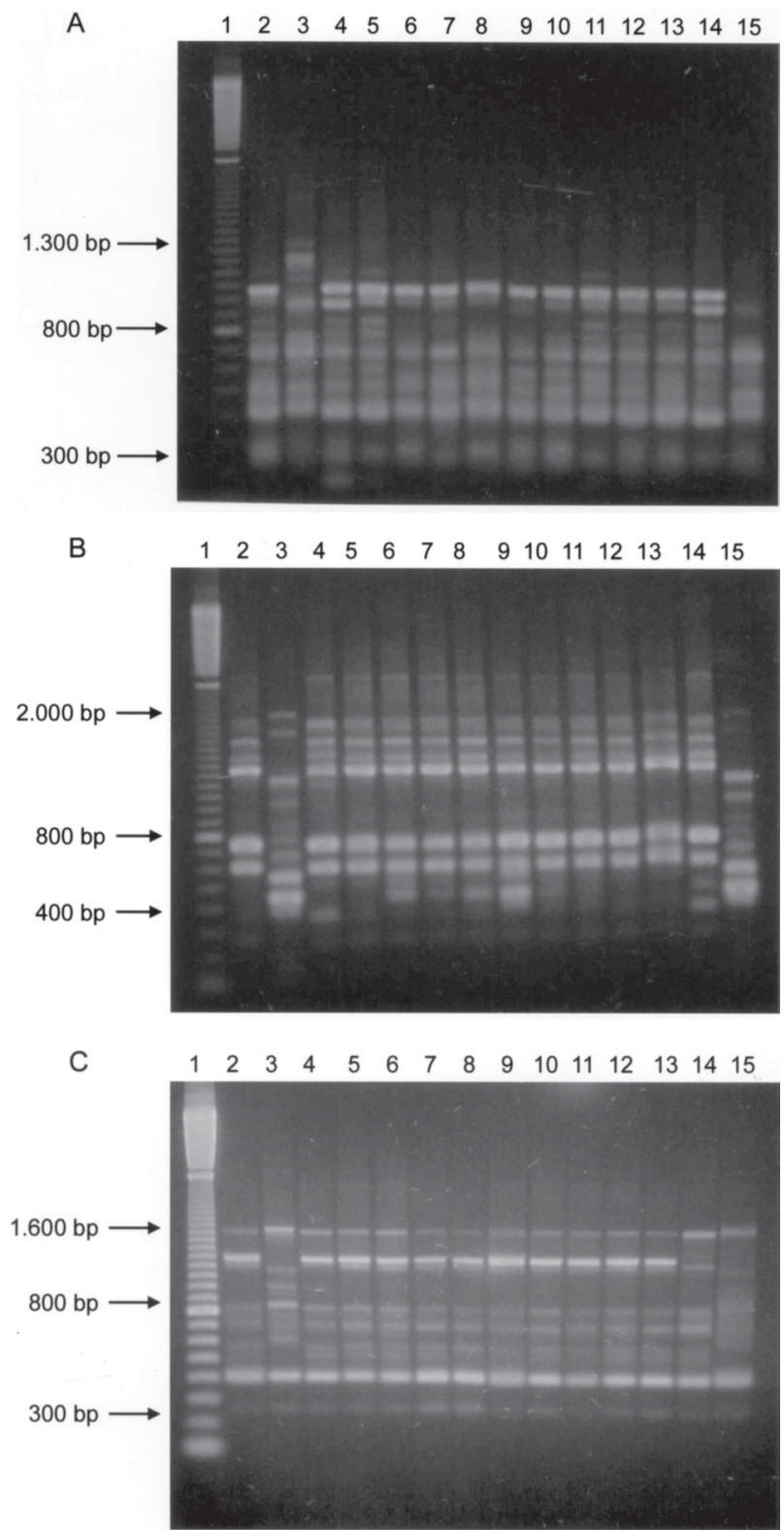

Fig. 1: agarose gels showing amplified DNA products generated using the CDU primer (A), B-14 primer (B) and the M2 primer (C), in RAPD analysis of Candida albicans and C. dubliniensis isolates. The profiles shown in lanes 2 and 3 correspond to the $C$. dubliniensis type strain CBS 7987 and the C. albicans reference strain ATCC 76615, respectively. Profiles shown in lanes 4-14 correspond to the $C$. dubliniensis isolates identified in this study, whereas profiles shown in lane 15 correspond to C. albicans isolate. Lane 1, molecular weight size reference markers (100 bp ladder).
2000 bp (Fig. 1B). Finally, using the primer M-2 we got 8 strong bands with $C$. dubliniensis and 5 with $C$. abicans isolates, with molecular sizes ranging from 300 to $1600 \mathrm{bp}$ (Fig. 1C). The other 35 isolates produced RAPD profiles very similar to that obtained with the $C$. albicans reference strain ATCC 76615.

All C. dubliniensis isolates belonged to serotype A. C. albicans isolates serotype A responded for $81.5 \%$ of all C. albicans strains. The distribution of C. albicans serotypes was very similar among isolates obtained from different clinical sources.

The 11 C. dubliniensis isolates exhibited MIC values ranging from 0.125 to 0.5 for amphotericin $\mathrm{B}, 0.06$ to 0.25 $\mu \mathrm{g} / \mathrm{ml}$ for fluconazole, 0.015 to $0.06 \mu \mathrm{g} / \mathrm{ml}$ for itraconazole and 0.015 to $0.03 \mu \mathrm{g} / \mathrm{ml}$ for ketoconazole. These data indicated that all of the $C$. dubliniensis isolates were susceptible to the 4 antifungal drugs tested. The range of the MIC values obtained with $C$. albicans isolates was larger than that obtained with $C$. dubliniensis isolates, as illustrated in Table I. It was possible to identify 17 C. albicans isolates resistant and/or with susceptibility dose dependent (SDD) to azoles, most of them (76\%) obtained from Aids patients. The MIC values exhibited by the azoles $\mathrm{SDD} /$ resistant isolates were presented in Table II.

\section{DISCUSSION}

In the present series we found a low prevalence rate of $C$. dubliniensis (2\%) among 548 yeast isolates obtained from a stock culture collection consisting of isolates that had been previously identified as C. albicans. The evaluated isolates were collected from different clinical sources of HIV positive and negative patients. As expected, the 11 C. dubliniensis isolates were obtained from oropharyngeal samples and 9 of them were cultured from HIV positive patients.

C. dubliniensis isolates have been mostly reported among Aids patients. The most extensive reported data of the incidence of $C$. dubliniensis in HIV infected and Aids patients comes from Ireland with a frequency about 18-32\% (Coleman et al. 1997, Sullivan et al. 1999). Studies conducted in the United States have reported oral incidence rates for $C$. dubliniensis in HIV infected individuals ranging from 11.1 to $17.5 \%$ (Kirkpatrick et al. 1998, Meiller et al. 1999). In South America, the prevalence of $C$. dubliniensis isolates seems to be lower than that exhibited in north hemisphere countries. A recent prospective and multicenter study conducted by Milan et al. (2001), including the evaluation of 108 Brazilian Aids patients with oropharyngeal candidiasis, found that only 3 of them were harboring $C$. dubliniensis at the time they were sampled. In accordance with the mentioned findings by Milan et al. (2001), other authors from Latin American medical centers have reported only limited number of Aids patients who were colonized and/or infected by $C$. dubliniensis (Sano et al. 2000, Alves et al. 2001).

The prevalence rate of $C$. dubliniensis found in our yeast stock collection was similar to that reported by similar studies performed by other investigators. Odds et al. (1998), using molecular typing for yeast identification, evaluated 2,589 isolates from stock cultures of yeasts originally identified as $C$. albicans and found that $2.1 \%$ of 
TABLE I

Antifungal susceptibility pattern of Candida albicans and Candida dubliniensis isolates determined by the NCCLS broth microdilution method

\begin{tabular}{llccc}
\hline Drug & Species/serotype (n) & MIC range $(\mu \mathrm{g} / \mathrm{ml})$ & $\mathrm{MIC}_{50}(\mu \mathrm{g} / \mathrm{ml})$ & $\mathrm{MIC}_{90}(\mu \mathrm{g} / \mathrm{ml})$ \\
\hline AMB & C. albicans/A (392) & $0.125-1.0$ & 0.5 & 0.5 \\
& C. albicans/B (91) & $0.125-1.0$ & 0.5 & 1.0 \\
& C. albicans - total (486) & $0.125-1.0$ & 0.5 & 0.5 \\
C. dubliniensis (11) & $0.125-0.5$ & 0.25 & 0.5 \\
FLZ & C. albicans/A (392) & $0.06-64$ & 0.25 & 0.5 \\
& C. albicans/B (91) & $0.125-64$ & 0.25 & 0.5 \\
& C. albicans - total (486) & $0.06-64$ & 0.25 & 0.25 \\
ITZ & C. dubliniensis (11) & $0.06-0.25$ & 0.125 & 0.06 \\
& C. albicans/A (392) & $0.007-4.0$ & 0.03 & 0.06 \\
& C. albicans/B (91) & $0.007-0.25$ & 0.03 & 0.06 \\
KTZ & C. albicans - total (486) & $0.007-4.0$ & 0.03 & 0.06 \\
& C. albicans/A (310) & $0.015-0.06$ & 0.03 & 0.06 \\
& C. albicans/B (73) & $0.007-8.0$ & 0.015 & 0.03 \\
& C. albicans - total (386) & $0.015-2.0$ & 0.015 & 0.06 \\
\end{tabular}

NCCLS: National Committee for Clinical Laboratory Standards; AMB: amphotericin B; FLZ: fluconazole; ITZ: itraconazole; KTZ: ketoconazole; MIC: minimal inhibitory concentration; $\mathrm{MIC}_{50}$ : $\mathrm{MIC}$ value able to inhibit $50 \%$ of the samples tested; $\mathrm{MIC}_{90}$ : $\mathrm{MIC}$ value able to inhibit $90 \%$ of the samples tested; $n$ : number of the isolates

\section{TABLE II}

Candida albicans isolates exhibiting resistance or susceptible dose-dependent to fluconazole, itraconazole and/or ketoconazole

\begin{tabular}{|c|c|c|c|c|c|}
\hline \multirow[b]{2}{*}{ Code } & \multirow[b]{2}{*}{ Source } & \multicolumn{4}{|c|}{$\mathrm{MIC}(\mathrm{mg} / \mathrm{ml})$} \\
\hline & & FLZ & ITZ & KTZ & Serotype \\
\hline A 30 & Oral cavities $^{a}$ & 64 & 4.0 & 4.0 & $\mathrm{~A}$ \\
\hline 1564 & Esophagus ${ }^{a}$ & 64 & 0.06 & 0.125 & $\mathrm{~A}$ \\
\hline 1173 & Urine & 64 & 0.015 & 4.0 & $\mathrm{~A}$ \\
\hline 447803 & Urine & 64 & 0.007 & 2 & $\mathrm{~B}$ \\
\hline 1542 & Esophagus $^{a}$ & 32 & 0.06 & 0.06 & $\mathrm{~A}$ \\
\hline 1446 & Esophagus ${ }^{a}$ & 16 & 0.5 & 0.125 & A \\
\hline 1416 & Esophagus ${ }^{a}$ & 16 & 0.25 & 0.125 & $\mathrm{~A}$ \\
\hline A13 & Oral cavities $^{a}$ & 8 & 0.25 & 0.125 & $\mathrm{~B}$ \\
\hline A64 & Oral cavities $^{a}$ & 2 & 0.125 & 0.5 & A \\
\hline $\mathrm{C} 20 \mathrm{~b}$ & Oral cavities & 2 & 0.06 & 0.25 & $\mathrm{~A}$ \\
\hline A16 & Oral cavities $^{a}$ & 1 & 0.06 & 1 & $\mathrm{~A}$ \\
\hline A98 & Oral cavities $^{a}$ & 1 & 0.015 & 1 & $\mathrm{~A}$ \\
\hline A 80 & Oral cavities $^{a}$ & 0.5 & 0.125 & 0.25 & $\mathrm{~A}$ \\
\hline 1407 & Esophagus $^{a}$ & 0.25 & 4 & 0.015 & A \\
\hline $112-\mathrm{H}$ & Blood & 0.25 & 0.25 & - & A \\
\hline A19 & Oral cavities $^{a}$ & 0.25 & 0.125 & 8 & A \\
\hline A 20 & Oral cavities $^{a}$ & 0.125 & 0.125 & 0.25 & A \\
\hline
\end{tabular}

MIC: minimal inhibitory concentration; FLZ: fluconazole; ITZ: itraconazole; KTZ: ketoconazole; $a$ : patient with Aids

them were correctly identified as $C$. dubliniensis. In a similar study performed by Jabra-Rizk et al. (2000) the prevalence of $C$. dubliniensis identification was $1.2 \%$ among the 1,251 isolates previously identified as C. albicans.

In the present study we found two $C$. dubliniensis isolates from oropharyngeal cultures obtained from HIV negative health carriers. These findings are in accordance with previous report of Coleman et al. (1997) that reported $3 \%$ of prevalence of this new species in health carriers from Ireland.

Isolation of $C$. dubliniensis from clinical sources other than oropharyngeal samples obtained from HIV negative patients have been reported with increasing frequency. C. dubliniensis isolates have been isolated from different clinical sources, including blood, urine, vaginal and feces from diabetic patients, surgical patients, and patients submitted to organ transplantation (Meis et al. 1999, Brandt et al. 2000, Willis et al. 2000).

Several methods for screening of $C$. dubliniensis have been reported, including culture on CHROMagar Candida, evaluation of their ability to grow at 42 or $45^{\circ} \mathrm{C}$, evaluation of $\beta$-D-glucosidase activity as well as their carbohydrate assimilation pattern (Sullivan et al. 1995, 1997, Schoofs et al. 1997, Pinjon et al. 1998, Salkin et al. 1998). Of note, as mentioned by other investigators, most of our cultures of $C$. dubliniensis that had been stocked by long periods of time and did not exhibited the typical dark green colony color on CHROMagar Candida medium when incubated up to 5 days (Schoofs et al. 1997, Sullivan et al. 1999). In our study, the inability of the organism to grow at high temperature $\left(42^{\circ} \mathrm{C}\right)$ was selected as a screening test for $C$. dubliniensis because it is inexpensive and easy to perform, generates results after $48 \mathrm{~h}$ incubation with a clear reading criteria and has been successfully used by different authors (Kirkpatrick et al. 1998, Tintelnot et al. 2000).

In the present study, the final identification of $C$. dubliniensis was obtained by RAPD analysis, a method standardized in our laboratory that has been used by several investigators as a molecular tool for the identification of microorganisms (Sullivan et al. 1995, Coleman et al. 1997, Melo et al. 1998, Park et al. 2000, Milan et al. 2001). It 
is noteworthy that the 3 different primers we used for RAPD analysis exhibited several high density bands that allowed a clear visual interpretation of the DNA fingerprinting exhibited by $C$. dubliniensis and C. albicans isolates.

All isolates of $C$. dubliniensis we found belonged to serotype A. The literature reports that $C$. dubliniensis isolates are able only to express serotype A phenotype of C. albicans (Schoofs et al. 1997, Sullivan \& Coleman 1998). Regarding to the antifungal susceptibility profile of the isolates, all them were susceptible to azoles and amphotericin B. Pfaller et al. (1999a), after evaluating a total of 71 C. dubliniensis isolates, found that most of them are highly susceptible to amphotericin B and azoles.

In Brazil, C. albicans serotype A susceptible to all antifungal drugs seems to be the most prevalent phenotype of C. albicans isolates, regardless of the origin of the sample. We found a frequency between $75.6 \%$ and $88.4 \%$ of serotype A among isolates obtained from HIV negative and positive, including all different clinical sources. This finding is in accordance with previous publications (Pires et al. 1996, Sant'Ana et al. 2002).

Despite the high susceptibility to azoles exhibited by the 486 C. albicans isolates, we found 17 (3.5\%) strains exhibiting susceptibility dose dependent status or resistance to azoles. It is noteworthy that 13 of the mentioned less susceptible isolates were recovered from Aids patients. C. albicans isolates resistant to fluconazole has been mainly reported among Aids patients with history of a long period of exposure to azoles (Persons et al. 1991, Millon et al. 1994, Barchiesi et al. 1996).

In conclusion, the clinical sources of $C$. dubliniensis in Brazil appears to be similar to that exhibited by isolates evaluated in other series of yeast stock cultures from $\mathrm{Eu}-$ rope and United States. Most of the isolates were obtained from oral cavities of HIV positive and Aids patients. C. albicans serotype A susceptible to all antifungal drugs was the major phenotype of C. albicans isolates stocked at our yeast collection, regardless of the clinical source of the strains.

\section{REFERENCES}

Alves SH, Milan EP, Branchini ML, Nishimura K, Fukushima K, Oliveira LO, Costa JM, Colombo AL 2001. First isolation of Candida dubliniensis in Rio Grande do Sul, Brazil. Diag Microbiol Infect Dis 39: 165-168.

Barchiesi F, Najvar LK, Luther MF, Scalise G, Rinaldi MG, Graybill JR 1996. Variation in fluconazole efficacy for Candida albicans strains sequentially isolated from oral cavities of patients with Aids in an experimental murine candidiasis model. Antimicrob Agents Chemother 40: 13171320.

Bauer D, Müller H, Reich J, Riedel H, Ahrenkiel V, Warthoe P, Strauss M 1993. Identification of differentially expressed mRNA tests by an improved display technique (DDRTPCR). Nucleic Acids Res 21: 4272-4280.

Brandt ME, Harrison LH, Pass M, Sofair NA, Huie S, Li R, Morrison CJ, Warnock DW, Hajjeh RA 2000. Candida dubliniensis fungemia: the first four cases in North America. Emerg Infect Dis 6: 46-49.

Coleman DC, Sullivan DJ, Bennett DE, Moran GP, Barry HJ, Shanley DB 1997. Candidiasis: the emergence of a novel species, Candida dubliniensis. Aids 11: 557-567.
Gales AC, Pfaller MA, Houston AK, Joly S, Sullivan DJ, Coleman DC, Soll DR 1999. Identification of Candida dubliniensis based on temperature and utilization of xilose and $\alpha$-methyl-D-glucoside as determined with the API 20C AUX and Vitek YBC sistems. J Clin Microbiol 37: 38043808 .

Jabra-Rizk MA, Falkler Jr. WA, Merz WG, Baqui AAMA, Kelley JI, Meiller TF 2000. Retrospective identification and characterization of Candida dubliniensis isolates among Candida albicans clinical laboratory isolates from human immunodeficiency virus (HIV)-infected and non-HIV-infected individuals. J Clin Microbiol 38: 2423-2426.

Kirkpatrick WR, Revankar SG, McAtee RK, Lopez-Ribot JL, Fothergill AW, McCarthy DI, Sanche SE, Cantu RA, Rinaldi MG, Patterson TR 1998. Detection of Candida dubliniensis in oropharyngeal samples from human immunodeficiency virus-infected patients in North America by primary CHROMagar Candida screening and susceptibility testing of isolates. J Clin Microbiol 36: 3007-3012.

Kurzai O, Heinz WJ, Sullivan DJ, Coleman DC, Frosch M, Mühlschlegel FA 1999. Rapid PCR test for discriminating between Candida albicans and Candida dubliniensis isolates using primers derived from the $\mathrm{pH}$-regulated $P H R 1$ and PHR2 genes of C. albicans. J Clin Microbiol 37: 1587 1590 .

Mannarelli BM, Kurtzman CP 1998. Rapid identification of Candida albicans and other human pathogenic yeasts by using short oligonucleotides in a PCR. J Clin Microbiol 36: 1634-1641.

Meiller TF, Jabra-Rizk MA, Baqui A, Kelley JI, Meeks VI, Merz WG, Falkler WA 1999. Oral Candida dubliniensis as a clinically important species in HIV-seropositive patients in the United States. Oral Surg Oral Med Oral Pathol Oral Radiol Endod 88: 573-580.

Meis JFGM, Ruhnke M, Pauw BED, Odds FC, Siegert W, Verweij PE 1999. Candida dubliniensis candidemia in patients with chemotherapy-induced neutropenia and bone marrow transplantation. Emerg Infect Dis 5: 150-153.

Melo ASA, Almeida LP, Colombo AL, Briones MRS 1998. Evolutionary distances and identification of Candida species in clinical isolates by Random Amplified Polymorphic DNA (RAPD). Mycopathologia 142: 57-66.

Milan EP, Burattini MN, Kalllas EG, Fischman O, Costa PRO, Colombo AL 1998. Azole resistance among oral Candida species isolates from Aids patients under ketoconazole exposure. Diagn Microbiol Infect Dis 32: 211-216.

Milan EP, Sant'Ana PL, Melo ASA, Sullivan DJ, Coleman DC, Lewi D, Colombo AL 2001. Multicenter prospective surveillance of oral Candida dubliniensis among adult Brazilian human immunodeficiency virus-positive and Aids patients. Diagn Microbiol Infect Dis 41: 29-35.

Millon L, Manteaux A, Reboux G, Drobacheff C, Monod M, Barale T, Michel-Briand Y 1994. Fluconazole-resistant recurrent oral candidiasis in human immunodeficiency viruspositive patients: persistence of Candida albicans strains with the same genotype. J Clin Microbiol 32: 1115-1118.

NCCLS-National Committee for Clinical Laboratory Standards 1997. Publication M27-A: Reference method for broth dilution antifungal susceptibility testing of yeasts. Approved Standard, Wayne PA. NCCLS 17: 1-28.

Odds FC, Nuffel LV, Dams G 1998. Prevalence of Candida dubliniensis isolates in a yeast stock collection. J Clin Microbiol 36: 2869-2873.

Park S, Wong M, Marras SAE, Cross EW, Kiehn TE, Chaturvedi V, Tyagi S, Perlin DS 2000. Rapid identification of Candida dubliniensis using a species-specific molecular beacon. $J$ Clin Microbiol 38: 2829-2836. 
Persons D, Laughlin M, Tanner D, Perfect J, Gockerman JP, Hathorn JW 1991. Fluconazole and Candida krusei fugemia. N Engl J Med 325: 1315.

Pfaller MA, Messer AS, Gee S, Joly S, Pujol C, Sullivan DJ, Coleman DC, Soll DR 1999. In vitro susceptibilities of Candida dubliniensis isolates tested against the new triazole and echinocandin antifungal agents. J Clin Microbiol 37: 870-872.

Pinjon E, Sullivan D, Salkin I, Shanley D, Coleman D 1998. Simple, inexpensive, reliable method for differentiation of Candida dubliniensis from Candida albicans. J Clin Microbiol 36: 2093-2095.

Pires MFC, Birman EG, Costa CR, Gambale W, Paula CR 1996. Candida albicans byotipes isolated from the oral cavity of HIV-positive patients. Rev Microbiol 27: 46-51.

Rodriguez-Tudella JL, Martinez-Suarez JV, Dronda F, Laguna F, Chaves F, Valencia E 1995. Correlation of in vitro susceptibility test results with clinical response: a study of azole therapy in Aids patients. $J$ Antimicrob Chemother 35: 793-804.

Salkin IF, Pruitt WR, Padhye AA, Sullivan D, Coleman D, Pincus DH 1998. Distinctive carbohydrate assimilation profiles used to identify the first clinical isolates of Candida dubliniensis recovered in the United States. J Clin Microbiol 36: 1467.

Sano A, Vilela MM, Takahashi I, Fukushima K, Takizawa K, Silva MT, Uno J, Nishimura K, Miyaji M 2000. Isolation of Candida dubliniensis from the oral cavity of an HIVpositive child in Brazil. Nippon Ishinkin Gakkai Zasshi 41: 177-181.

Sant'Ana PL, Milan EP, Martinez R, Queiroz-Telles F, Ferreira MS, Alcântara AP, Carvalho MT, Colombo AL 2002. Multicenter Brazilian study of oral Candida species isolated from Aids patients. Mem Inst Oswaldo Cruz 97: 253257.

Schoofs A, Odds FC, Colebunders R, Ieven M, Goossens H 1997. Use of a specialization isolation media for recognition and identification of Candida dubliniensis isolates from HIVinfected patients. Eur J Clin Microbiol Dis 16: 296-300.

Schorling SR, Kortinga HC, Froschb M, Mühlschlegel FA 2000. The role of Candida dubliniensis in oral candidiasis in human immunodeficiency virus-infected individuals. Crit Rev Microbiol 26: 59-68.

St-Germain G, Dion C, Espinel-Ingroff A, Ratelle J, Repentigny L 1995. Ketoconazole and itraconazole susceptibility of Candida albicans isolated from patients infected with HIV. J Antimicrob Chemother 36: 109-118.

Sullivan D, Coleman D 1998. Candida dubliniensis: characteristics and identification. J Clin Microbiol 36: 329-334.

Sullivan DJ, Haynes K, Bille J, Boerlin P, Rodero L, Lloyd S, Henman M, Coleman D 1997. Widespread geographic distribution of oral Candida dubliniensis strains in human immunodeficiency virus-infected individuals. J Clin Microbiol 35: 960-964.

Sullivan DJ, Westerneng TJ, Haynes KA, Bennett DE, Coleman DC 1995. Candida dubliniensis sp. nov. phenotypic and molecular characterization of a novel species associated with oral candidosis in HIV infected individuals. Microbiology 141: 1507-1521.

Sullivan DJ, Moran G, Donnelly S, Gee S, Pinjon E, McCartan B, Shanley DB, Coleman DC 1999. Candida dubliniensis: an update. Rev Iberoam Micol 16: 72-76.

Tintelnot K, Haase G, Seibold M, Bergmann F, StaemmLer M, Franz T, Naumann D 2000. Evaluation of phenotypic markers for selection and identification of Candida dubliniensis. J Clin Microbiol 38: 1599-1608.

Wach A, Pich H, Phillipsen P 1994. Procedures of isolating yeast DNA for different purposes. In JR Johnston, Molecular Genetics of Yeast, Oxford University Press, Oxford, p. 10-11.

Willis AM, Coulter WA, Sullivan DJ, Coleman DC, Hayes JR, Bell PM, Lamey PJ 2000. Isolation of Candida dubliniensis from insulin-using diabetes mellitus patients. J Oral Pathol Med 29: 86-90. 